\title{
Saturation of the Lactate Clearance Mechanisms Different from the "Lactate Shuttle" Determines the Anaerobic Threshold: Prediction from the Bioenergetic Model
}

\author{
Tiziano Binzoni ${ }^{1), 2)}$ \\ 1) Department of Radiology, Geneva University Hospital, Geneva, Switzerland \\ 2) Department of Physiology, University of Geneva, Geneva, Switzerland
}

\begin{abstract}
It is demonstrated, that the bioenergetic model combined with the mathematical constraints determined by the experimental knowledge of the aerobic metabolism and the Lohmann reaction dictates the exact lactate (La)-time relationship during exercise. The theory predicts that $\mathrm{La}$ is necessarily produced (above the resting baseline), even during extremely low work loads, where the metabolism was usually considered in the past to be "pure" aerobic. The La rate of production increases linearly as a function of the work load. The anaerobic threshold is strictly determined by the saturation of the La clearance mechanisms of the body different from the "La shuttle" and not by the involvement of a sudden increased La production at the cellular level. These results imply that the half time of the PCr breakdown kinetics at the onset of a constant load exercise can be expressed as a function of the onset speed of the aerobic and of the anaerobic metabolism, even in the case of a very low mechanical power. The PCr halftime does not depend on the workload and represents a physiological invariant. The bioenergetic model was created during a long historical period, when it was believed that the La production was not present at all for very low exercise levels but, actually, the bioenergetic model predicts exactly the opposite result! J Physiol Anthropol Appl Human Sci 24 (2): 175-182, 2005 http://www.jstage.jst.go.jp/browse/jpa
\end{abstract}

[DOI: $10.2114 /$ jpa.24.175]

Keywords: anaerobic threshold, skeletal muscle, human, lactate production

\section{Introduction}

The rules governing lactate $(\mathrm{La})$ production or utilization during exercise have always been a matter of animate debates (Myers and Ashely, 1997). The reasons underlying these scientific diatribes may be found in part in the technical difficulties encountered when trying to assess the time course of the intracellular La concentration and, in particular, when dealing with human investigations. In fact, intracellular $\mathrm{La}$ concentrations are often indirectly estimated from the analysis of blood samples or, at the tissue level, by muscle biopsies (Knuttgen and Saltin, 1972). Nuclear magnetic resonance spectroscopy is also a powerful tool allowing the investigators to assess intramuscular La concentration (Kemp and Radda, 1994). However, all these techniques do not allow us to distinguish between $\mathrm{La}$ production and utilization, because they yield only total La concentrations in the tissue or blood. In spite of these technical problems, the experimental approach based on blood La measurements led to important concepts as the "anaerobic threshold" or the "oxygen debt" (for a review see: di Prampero, 1981). On the other side, the introduction of the isotope labelling technique allowed several investigators to make a more precise distinction between La appearance and desappearance, at rest and during exercise, and this generated the idea of "lactate shuttle" (for a review see: Brooks, 2000; Brooks, 2002).

In spite of these technical improvements and the possibility to obtain more detailed analyses of the La behaviour during exercise, the experimental data could not be organized into one and the same coherent picture and the believer of the "anaerobic threshold" or of the "lactate shuttle" started having animated discussions. In fact, Brooks in his review wrote: [...the "lactate shuttle" hypothesis did not find wide acceptance because it made no sense to those accepting classic "oxygen debt" or "anaerobic threshold" theories] (Brooks, 2000). Nowadays, all these concepts and experimental data are relatively well accepted. However, no explicit mathematical model has yet been formulated. Thus, in the present paper we will attempt to propose a mathematical approach dealing with the concept of "anaerobic threshold" and with La production during a so called "pure" aerobic exercise.

For explanatory reasons, in the following sections the energy needs imposed by an exercise will be defined as equal to the total energy demand minus the energy demand coming from 
the resting metabolic activity i.e., the resting energy demand will be methodically considered as a baseline. For La production we will intend only the La actually produced by the exercise, while any other La clearence mecanism will be treated mathematically with a separated term and definition (i.e., the La one can observe in the blood will in this case be explained as the combined result of "production" and "clearance"). The mathematical results presented in this paper, have been derived from the so called bioenergetic model (di Prampero, 1981; Binzoni, 2003) and based only on few and very well known physiological observations, independently of any La measurements.

In the present work, it is predicted that La must be always produced, even during the classical so defined "pure" aerobic exercise and also even if we take into account the "La shuttle", a fact, the latter, which nowadays is also supported experimentally (Brooks, 2002). Moreover, it is concluded that there is no sudden change in the rate of La production for a given critical level of mechanical work (a power usually corresponding to the "anaerobic threshold") and thus that the "anaerobic threshold" is only determined by the La clearance mechanisms different from the "La shuttle". It must be noted, that the bioenergetic model was created during a long historical period when it was believed that La production did occur at all for very low exercise levels (di Prampero, 1981). The curious fact is that this model predicts exactly the opposite result! The strong believe in the existence of "pure" aerobic exercises led to mathematical "over-simplifications" hindering the real sense of the model. We hope, that this work will help to finally reconcile the "old" with the "new" school.

\section{Theory}

\section{The bioenergetic model}

Muscle energetics at rest or during exercise, can be described taking into account three main biochemical systems: the aerobic glycolysis, the anaerobic glysolysis and the Lohmann reaction (di Prampero, 1981). These three biochemical systems produce the ATP necessary for the muscle activity. It has been demonstrated over the past 70 years that this complex biochemical network may be described globally by means of the bioenergetic model as (Binzoni, 2003):

$$
\begin{aligned}
\dot{E}_{\text {work }}+\dot{\mathrm{E}}_{\text {rest }}= & {[\mathrm{AT} \dot{\mathrm{TP}}]+\alpha[\mathrm{P} \dot{\mathrm{C}}]+\beta\left([\dot{\mathrm{La}}]+[\dot{\mathrm{La}}]_{\mathrm{rest}}\right) } \\
& +\gamma\left(\left[\dot{\mathrm{O}}_{2}\right]+\left[\dot{\mathrm{O}}_{2}\right]_{\mathrm{rest}}\right)
\end{aligned}
$$

where $\dot{E}_{\text {work }}$ (the dot is, as usual, the time derivative) represents the instantaneous energy consumption of the muscle per unit time (in ATP concentration units, $\mathrm{mM} \mathrm{s}^{-1}$ ) and [ATP], [PCr], [La] and $\left[\mathrm{O}_{2}\right]$ are the concentrations of ATP, phosphocreatine $(\mathrm{PCr})$, lactate $(\mathrm{La})$ and oxygen $\left(\mathrm{O}_{2}\right)$, respectively. The term $\dot{\mathrm{E}}_{\text {rest }}=\beta[\mathrm{La}]_{\text {rest }}+\gamma\left[\dot{\mathrm{O}}_{2}\right]_{\text {rest }}$ represents the resting metabolism. The first term on the right hand side of Eq. (1), contributes only in extreme conditions, when the muscle cannot succeed in maintaining a constant ATP concentration (e.g., fatigue). $\alpha, \beta$ and $\gamma$ represent the number of ATP molecules produced by one molecule of $[\mathrm{PCr}],[\mathrm{La}]$ and $\left[\mathrm{O}_{2}\right]$, respectively. The terms, $\alpha[\mathrm{PCr}], \beta[\mathrm{La}]$ and $\gamma\left[\mathrm{O}_{2}\right]$ yield the overall amount of ATP produced by the Lohmann reaction, the anaerobic glycolysis and the aerobic glycolysis, respectively. Eq. (1) has the advantage to describe the energy balance of the working muscle without the need of explicitly express the single energy stores (e.g., glycogen stores, lipids stores, etc.) and it implicitly contains all the classical pathways (e.g., the Krebs cycle, the beta-oxidation, etc) (di Prampero, 1981).

From now on, the working hypothesis is that the model represented by Eq. (1) is of course true; the aim being to see which are the physiological predictions that we can obtain from the model in the domain of the anaerobic metabolism and this, without using experimental results concerning the $\mathrm{La}$ metabolism. Then, in the Dicussion section we will analyse the predictions in the light of the known scientific literature. The analytical (symbolic) form of the results will allow one to obtain the general behaviour of the different mechanisms (e.g., constant, linear, exponential, etc.) without the need to utilise numerical quantities that are sometimes difficult to measure experimentally and/or have a large variability.

At this point, Eq. (1) can be further simplified for the present purposes. The efficiency $(\eta)$ of the energy utilization for a given mechanical work may be defined as:

$$
\eta \equiv \frac{\dot{w}}{\dot{E}_{\text {work }}}
$$

where $\dot{w}$ represents the mechanical work $\left(\mathrm{J} \mathrm{s}^{-1}\right)$. From Eqs. (1) and (2) one obtains:

$$
\frac{\dot{w}}{\eta}=[\mathrm{ATP}]+\alpha[\mathrm{PC} \mathrm{r}]+\beta[\dot{\mathrm{La}}]+\gamma\left[\dot{\mathrm{O}}_{2}\right]
$$

where the term $\dot{\mathrm{E}}_{\text {rest }}=\beta\left[\mathrm{La}_{\text {rest }}+\gamma\left[\dot{\mathrm{O}}_{2}\right]_{\text {rest }}\right.$ has been substracted. So, Eq. (3) describes the net energy expenditure during exercise without the resting metabolism. It is essential to notice that Eq. (3) describes the metabolism directly at the cellular level. In fact, the model takes into account, globally, the contribution of each single muscle cell and the different ATP rates produced by the biochemical pathways, starting from different energy stores (e.g., lipids, glycogen, etc.), are implicitly expressed (as a total contribution) in the parameters $\alpha, \beta$ and $\gamma$. An intuitive explanation of Eq. (3) may be obtained from Fig. 1. Based on Fig. 1 (summary of a set of figures derived from di Prampero et al. (1998)) we will shortely discuss Eq. (3) and, in particular, the less trivial $\gamma\left[\dot{\mathrm{O}}_{2}\right]$ and $\beta[\mathrm{La}]$ terms. In Fig. 1 , the indexes $i$ referes to the " $i$-th" cell (muscle fibre) and we suppose that the investigated muscle is composed by $N$ cells (i.e., Eq. (3) describes the total ATP production of these $N$ cells). Let us before consider the cell " $i$ ". As it is well known, all the main substrates are transformed in pyruvate ( $\mathrm{Pa}$, in Fig. 1) along the path. 


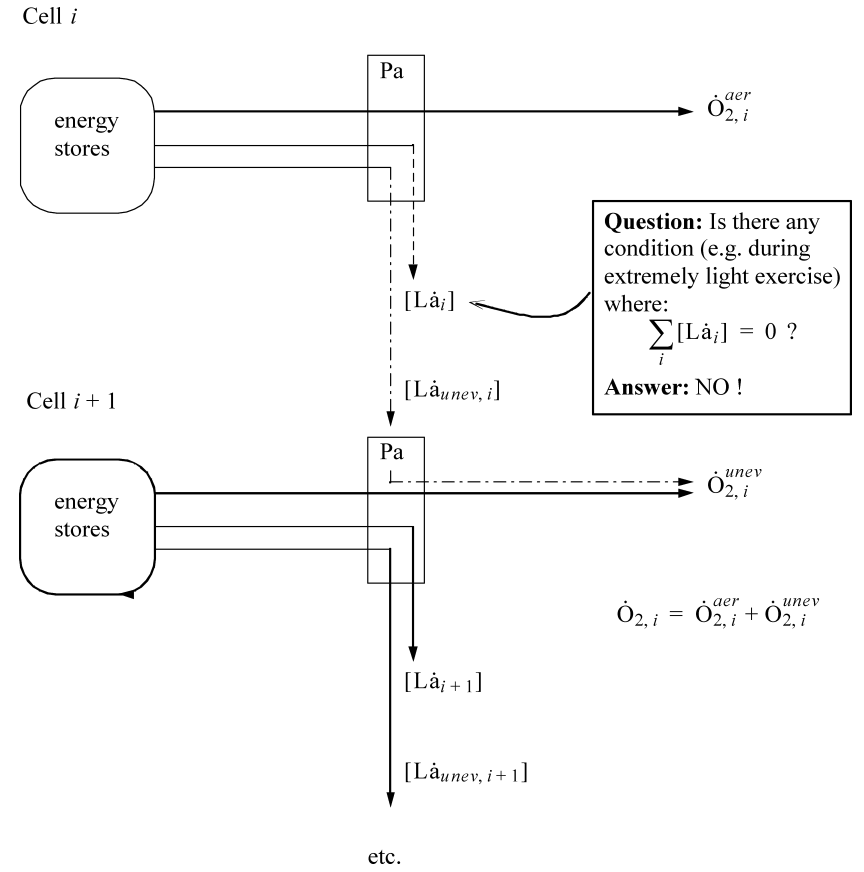

Fig. 1 Representation of the metabolic biochemical pathways generating ATP during exercise (see text).

The pathway (cell $i$ ) going from "energy stores" to $\left[\dot{\mathrm{O}}_{2, i}^{a e r}\right]$ (continuous line), represents in this case a pure aerobic metabolism, i.e. the subtrate is completely oxidated to $\mathrm{CO}_{2}$ and $\mathrm{H}_{2} \mathrm{O}$, and the cell $i$ uses oxygen at the rate $\left[\dot{\mathrm{O}}_{2, \mathrm{i}}^{a e r}\right]$. In this case, the number of ATP moles produced per mole of $\mathrm{O}_{2}$ for the $i$ cell is by definition, $\gamma_{i}^{a e r}\left[\dot{\mathrm{O}}_{2, \mathrm{i}}^{\text {aer }}\right]$. We are obliged to define the constant $\gamma_{i}^{a e r}$ using an index $i$ because different cells may utilize slightly different subtrates (e.g. glycogen, lipids, or both, etc). Now, one can express the ATP production in term of total $\mathrm{O}_{2}$ consumption as: $\gamma_{i}^{\text {aer }}\left[\dot{\mathrm{O}}_{2, \mathrm{i}}^{\text {aer }}\right]=\gamma_{i}^{\text {aer }} f_{i}^{\text {aer }}\left[\dot{\mathrm{O}}_{2}\right]$, where $f_{i}^{a e r}$ is the fraction of $\mathrm{O}_{2}$ utilized by the $i$-th cell for the "continuous line" pathway. Of course, $\left[\dot{\mathrm{O}}_{2}\right]$ is the total $\mathrm{O}_{2}$ consumption appearing in Eqn. (3).

The "dash-dot" pathway, represents a typical situation where the cell produces $\mathrm{La}\left(\left[\mathrm{La}_{\text {unev, }, i}\right]\right.$ in Fig. 1$)$ and this $\mathrm{La}$ is then "recycled" e.g. by another cell (e.g., the cell $i+1$ in Fig. 1) after transforming La again in pyruvate. This, metabolism has been defined as "unevenly aerobic" (di Prampero et al., 1998) and needs also the utilization of $\mathrm{O}_{2}$. In any case, if one observe the "dash-dot" pathway, we realize that in term of ATP production is equivalent to the "continuous line" pathway (e.g., it follows the same biochemical steps). For this reason, the ATP production rate for the "dash-dot" pathway can be written also in this case as: $\gamma_{i}^{\text {unev }} \dot{\mathrm{O}}_{2, i}^{\text {unev }}=\gamma_{i}^{\text {unev }} f_{i}^{\text {unev }}\left[\dot{\mathrm{O}}_{2}\right]$, with analogous definition as for the "continuous line" pathway. This term takes also into account the so called "La shuttle" mechanism, where the La produced by one cell is used by another cell.

Thus, the total ATP rate of the muscle ( $N$ cells) generated by the "continuous line" ("evenly aerobic") and "dash-dot" ("unevenly aerobic") biochemical pathways is:

$$
\begin{aligned}
\gamma\left[\dot{\mathrm{O}}_{2}\right] & =\sum_{i=1}^{N}\left\{\gamma_{i}^{\text {aer }} f_{i}^{\text {aer }}\left[\dot{\mathrm{O}}_{2}\right]+\gamma_{i}^{\text {unev }} f_{i}^{\text {unev }}\left[\dot{\mathrm{O}}_{2}\right]\right\} \\
& =\sum_{i=1}^{N}\left\{\gamma_{i}^{\text {aer }} f_{i}^{\text {aer }}+\gamma_{i}^{\text {unev }} f_{i}^{\text {unev }}\right\}\left[\dot{\mathrm{O}}_{2}\right]
\end{aligned}
$$

where $\gamma$ has been defines as:

$$
\gamma \equiv \sum_{i=1}^{N}\left\{\gamma_{i}^{\text {aer }} f_{i}^{a e r}+\gamma_{i}^{\text {unev }} f_{i}^{\text {unev }}\right\}
$$

The term $\gamma\left[\dot{\mathrm{O}}_{2}\right]$ is the term appearing in Eq. (3) and, thanks to the parameter $\gamma$, it takes globally into account any possible substrate utilization and/or metabolic differences existing between the cells (e.g., fast twich fibres, slow twich fibres, etc). In practice, in humans one can usually obtain experimental informations only on this "global" $\gamma$ parameter because the "single cell" access is practically impossible during exercise protocols. In the present context, we define $\gamma\left[\dot{\mathrm{O}}_{2}\right]$ to be the "aerobic metabolism".

The remaining pathway, is the "dashed" (Fig. 1) pathway and it represents the "anaerobic metabolism" where we have La formation during ATP production. This La is not "recycled" and it represents for example the La that is mesurable in the blood. Thus, using a similar procedure as above, the total ATP rate (whole muscle) produced by the "dashed" pathway can be easily defined as:

$$
\beta[\dot{\mathrm{La}}]=\sum_{i=1}^{N} \beta_{i}\left[\dot{\mathrm{La}_{i}}\right]=\sum_{i=1}^{N} \beta_{i} f_{i}^{\mathrm{La}}[\dot{\mathrm{La}}]
$$

where $f_{i}^{\mathrm{La}}$ is the fraction of the total La production for the cell $i$ and:

$$
\beta \equiv \sum_{i=1}^{N} \beta_{i} f_{i}^{\mathrm{La}}
$$

Thus, in the present work, the term $\beta\left[\mathrm{La}^{\circ}\right]$ represents the "anaerobic metabolism". The remaining terms appearing in Eq. (3) are trivial and will not be discussed here. After having clarified the intuitive "meaning" of Eq. (3) let one carry on on the mathematical analysis.

The La production at steady state is never nil and increases linearly with work load

We will now analyse an exercise at steady state, where the mechanical power is situated anywhere between the rest and the maximal oxygen consumption (i.e. in the range where the oxygen consumption is linear as a function of the mechanical power, see below) and [La] below the maximum rate of production (Margaria et al., 1964). No conditions are imposed on the La concentration behaviour inside this metabolic range. The above exercise protocol impose a supplementary constraint on the remaining terms (i.e., not lactic) of Eq. (3). In 
fact, the terms $[\mathrm{AT} P]$ and $[\mathrm{PC} r]$ may be reasonably neglected because the corresponding concentrations remains constants at steady state (Ross et al., 1982). Thus, Eq. (3) may be simplified as:

$$
\frac{\dot{w}}{\eta} \approx \beta\left[\mathrm{La}_{s s}\right]+\gamma\left[\dot{\mathrm{O}}_{2 s s}\right]
$$

where the index ss means at steady state. Considered that below the maximal oxygen consumption $\left[\dot{\mathrm{O}}_{2 s s}\right]$ is linearly related to $\dot{w}$, then Eq. (8) becomes:

$$
\frac{\dot{w}}{\eta}=\beta\left[\dot{\mathrm{a}}_{s s}\right]+\gamma \gamma_{w} \dot{w}
$$

Analytically, Eq. (9) has been obtained from Eq. (8) by assuming that $\left[\dot{\mathrm{O}}_{2 s s}\right]=\gamma_{w} \dot{w}$, where $\gamma_{w}$ is the proportionality constant describing the number of $\mathrm{O}_{2}$ molecules per second required to develop a given power $\dot{w}$.

Equation (9) imposes a strong constraint on $\left[\mathrm{La}_{s s}\right]$ behaviour and it necessarily implies that $\beta\left[\mathrm{La}_{s s}\right]$ as a function of $\dot{w}$ is linear. This result derives directly (mathematically) from the bioenergetic model without the need of any knowledge on La production during exercise.

Fig. 2 schematically shows Eq. (9) and stress another interesting fact: $\beta\left[\dot{\mathrm{a}}_{s s}\right]$ (and thus $\left[\dot{\mathrm{L}}_{s s}\right]$ ) can be nil only for $\dot{w}=0 \mathrm{~W}$ ! In fact, above the "anaerobic threshold" $\beta\left[\dot{\mathrm{La}}_{s s}\right] \neq 0$ by definition (and experimentally) and thus, due to the linearity (demonstrated above), also for any $\dot{w}>0$.

Indeed, Eq. (9) can be written as:

$$
\left[\dot{\mathrm{L}}_{s s}\right]=\frac{1}{\beta}\left(\frac{1}{\eta}-\gamma_{w}\right) \dot{w}
$$

which shows that the slope for the $\left[\dot{\mathrm{a}}_{s s}\right]-\dot{w}$ relationship is the constant $\beta^{-1}\left(\eta^{-1}-\gamma \gamma_{w}\right)$. This constant is non nil and thus, by definition, the following condition applies:

$$
\left(\frac{1}{\eta}-\gamma_{w}\right)>0
$$

This defines a precise range of values for $\eta, \gamma$ and $\gamma_{w}$ and imposes the constraint that the efficiency $\eta$ can never be greater than $\left(\gamma \gamma_{w}\right)^{-1}$.

The above considerations imply that $\left[\dot{\mathrm{a}}_{s s}\right]$ can also be written as:

$$
\left[\mathrm{L} \dot{\mathrm{a}}_{s s}\right]=\beta_{w} \dot{w}
$$

where $\beta_{w} \equiv \beta^{-1}\left(\eta^{-1}-\gamma \gamma_{w}\right)$ is positive and represents the proportionality constant describing the number of $\mathrm{La}$ molecules necessary to develop a given amount of work. Eq. (12) means that $\left[\dot{\mathrm{L}}_{s s}\right]$ (La production) can never be zero even below the anaerobic threshold. Only when $\dot{w}=0$ the lactate produced by the muscle is zero. It is useful to stress again that we do not consider here the resting metabolism and that Eq.

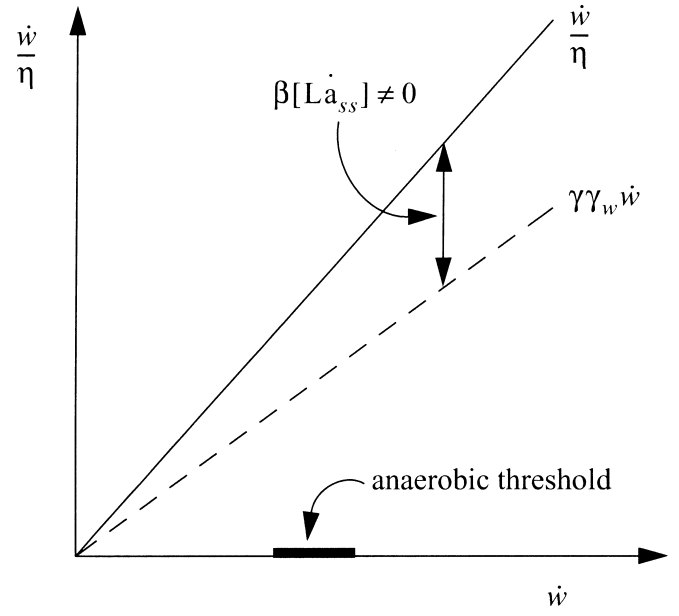

Fig. 2 Total energy expenditure per unit time $(\dot{w} / \eta)$ at steady state, as a function of the mechanical work load $(\dot{w})$. The dashed line represents the aerobic contribution $\left(\gamma\left[\dot{\mathrm{O}}_{2 s s}\right]=\gamma \gamma_{w} \dot{w}\right)$. At $\dot{w}=0$, both the aerobic and anaerobic contributions must be nil. Above the anaerobic threshold (black horizontal bar) the anaerobic contribution $\left(\beta\left[\mathrm{La}_{s s}\right]\right)$ is, by definition, non nil. This constraints implies that the two linear functions may never be superposed, so the anaerobic contribution to the total energy for any $\dot{w}$ value, must be $>0$.

(12) holds in the metabolic range were [نa] is below the maximum rate of La production (Margaria et al., 1968) and were $\left[\dot{\mathrm{O}}_{2 s s}\right]$ is linearly related to $\dot{w}$. For completeness, Eq. (10) and Eq. (12) allow us to write the exact expression for $\eta$ :

$$
\eta=\frac{1}{\beta \beta_{w}+\gamma \gamma_{w}}
$$

\section{Discussion}

\section{The steady state condition}

It has been shown that the bioenergetic model, combined with the well known physiological observations, that: 1) the oxygen consumption is linearly related to the mechanical power and; 2) the $\mathrm{PCr}$ consumption is nil at steady state; lead to the mandatory conclusion (mathematically derived from the model) that the lactate production can never be nil, even in the case of a very moderate aerobic exercise (see Eq. (12)) and that the rate of production is linearly related to the mechanical power $(\dot{w})$. It is of notice, that this result was obtained without the need of introducing any experimental information concerning the lactate production during exercise, as it is usually necessary with other approaches, e.g. in the case of metabolic control theories were an activation function has to be defined (Mader and Heck, 1986). As a consequence, the hypothesis stating that "it exists a range of work loads where the metabolism is totally aerobic", is wrong (again we do not consider here the La production at rest). This hypothesis can at best be considered only as a good approximation for very low work levels. The experimental results obtained e.g. on isolated 
animal tissues (Connett et al., 1984) or humans (Stanley et al., 1986), where it was demonstrated that $\mathrm{La}$ is produced even at very low muscle stimulation levels, further support the present findings.

So, if La is always produced at steady state (Fig. 1 and Eq. (12)), it remains to be explained how the "anaerobic threshold" may be observed in the blood and/or tissue. In fact, Eq. (12) describes only the La produced by the muscle but does not explain what happens in the blood/tissue. Thus, mathematically, if one want to modify Eq. (12) to hold for the blood/tissue, and obtain a zero net La accumulation below the anaerobic threshold and in presence of continuous $\mathrm{La}$ production from the muscle, we must substract a term on the right hand side of Eq. (12) (see below). This is the only mathematical solution. Intuitively, this is physiologically equivalent to eliminate the La using other mechanisms, such as the resynthesis performed by tissues such the liver, heart, kidney, etc. (see e.g., Antonutto and di Prampero, 1995; Brooks, 2000; Brooks, 2002). These La clearing mechanisms must not use $\mathrm{O}_{2}$, because the $\mathrm{O}_{2}$ consuming mechanisms have already been taken into account in the $\gamma_{i}^{\text {unev }} \dot{\mathrm{O}}_{2, i}^{\text {unev }}$ terms implicit in $\gamma \dot{\mathrm{O}}_{2}$. This network of "clearing" mechanisms must have, by definition, a global biological saturation level or a finite capacity to eliminate the La produced by the working muscle cells. When $\dot{w}$ is increased, a value must be reached, defined here as the "anaerobic threshold", where the system is no more able to increase the rate of La elimination. At this point production exceeds elimination and the net La concentration starts to increase in the blood/tissue. To summarize, if one wants a zero La production in the blood/tissue and then a "threshold" for a given $\dot{w}$, the substraction of a new term in Eq. (12) is the only mathematical solution.

Actually, the anaerobic threshold can not be a very precise $\dot{w}$ value, but for explanatory reasons lets admit it is so. If it is the case, the increase of La concentration in the blood/tissue as a function of time, $\left[\Delta \mathrm{La}_{\text {tissure }}\right]$, can be described with the help of Eq. (12) as:

$$
\left[\Delta \mathrm{La}_{\text {tissue }}\right] \equiv \int_{t_{0}}^{t}\left(\left[\dot{\mathrm{La}}_{s s}\right]-\left[\dot{\mathrm{La}}_{\text {Clear }}\right]\right) d t^{\prime}
$$

where $\left[\mathrm{La}_{\text {Clear }}\right]$ is the clearance rate for the lactate (the new term we want to substract to Eq. (12)), $t_{0} \geq t_{\text {steady }}$ is any time at steady state and $t_{\text {steady }}$ is the minimum time necessary to reach the steady state. Then, the integral of both sides of the modified Eq. (12) is taken. The integral describes of course the changes in La concentration (or the net accumulation) as a function of time (for $t \geq t_{0}$ ). Within the above definitions, $\left[\mathrm{La}_{\text {Clear }}\right]$ can be expressed as:

$$
\left[\dot{\mathrm{La}}_{\text {Clear }}\right]= \begin{cases}{\left[\dot{\mathrm{La}}_{\text {ss }}\right],} & \dot{w}<\dot{w}_{\text {threshold }} \\ {\left[\dot{\mathrm{La}}_{\text {MaxClear }}\right],} & \dot{w} \geq \dot{w}_{\text {threshold }}\end{cases}
$$

where $\left[\mathrm{La}_{\text {MaxClear }}\right]$ is the maximal $\mathrm{La}$ clearence rate (assumed to be constant) above the anaerobic threshold and $\dot{w}_{\text {threshold }}$ represents the mechanical power at which the anaerobic threshold is reached. Below the anaerobic threshold $\left[\mathrm{La}_{\text {Clear }}\right]=\left[\mathrm{La}_{s s}\right]$; thus production and clearance are perfectly balanced and therefore $\left[\Delta \mathrm{La}_{\text {tissue }}\right]=0$ (i.e., no net $\mathrm{La}$ increase in tissue/blood). It must be highlighted again the fundamental point that Equation (14) does not describe the transient phase, when $t<t_{0}$. Actually, during this phase $\left[\mathrm{La}_{\text {Clear }}\right]$ might be different (smaller) than [ $\left.\mathrm{La}^{\circ}\right]$, and thus when the steady state is reached, the La concentration in blood and tissues might be higher than that prevailing at rest ("early lactate", di Prampero et al., 1970).

If $\left[\dot{L a}_{s s}\right]$ is known for $\dot{w} \geq \dot{w}_{\text {threshold }}$ (Eq. (12)), by intergration of Eq. (14) one obtains that at steady state:

$$
\begin{aligned}
{\left[\Delta \mathrm{La}_{\text {tissue }}\right] } & =\int_{t_{0}}^{t}\left(\beta_{w} \dot{w}-\left[\dot{\mathrm{La}}_{\text {MaxClear }}\right]\right) d t^{\prime} \\
& =\left(\beta_{w} \dot{w}-\left[\dot{\mathrm{La}}_{\text {MaxClear }}\right]\right)\left(t-t_{0}\right)
\end{aligned}
$$

Thus, above the anaerobic threshold, the tissue $\mathrm{La}$ concentration, $\left[\Delta \mathrm{La}_{\text {tissue }}\right]$, increases linearly as a function of time (for a given power) or as a function of the mechanical power (for a given time). Eq. (16) holds for $\left[\mathrm{La}_{\mathrm{ss}}\right]=$ $\beta_{w} \dot{w} \geq\left[\mathrm{La}_{\text {MaxClear }}\right]$; below this value $\left[\Delta \mathrm{La}_{\text {tissue }}\right]=0$ (see Eq. (15)) i.e., the La concentration in the tissue remains constant at the value reached at the end of the transient phase. This behaviour is compatible with "classical" experimental findings obtained on the treadmill (Margaria et al., 1964) (where it was assumed that blood $\mathrm{La}$ is representative of the body average $\mathrm{La}$ concentration).

It has been observed that training does not change the $\left[\dot{\mathrm{O}}_{2}\right]-\dot{w}$ relationship (we mean here the "linear" part of $\left[\dot{\mathrm{O}}_{2}\right]-\dot{w}$, in the $\dot{w}$ range defined in the section "The La production is never nil and increases linearly with work load") and that the mechanical efficiency may be considered reasonably unchanged. If this is the case, then the present mathematical derivation (Eq. (9), Eq. (12) and Fig. 2) implies that $\beta_{w}$ is not affected by training. In this context (training), it has also been observed that the $\left[\Delta \mathrm{La}_{\text {tissue }}\right]-\dot{w}$ relationship (for a given $t$ ) is shifted to the right i.e., the anaerobic threshold, $\dot{w}_{\text {threshold }}$, is increased. Thus, it can be concluded, by inspecting Eq. (16), that $\left[\mathrm{La}_{\text {MaxClear }}\right]$ must be increased. This result corroborate the experimental finding that training seems to increase lactate metabolic clearance (Fukuba, 1992; Stallknecht et al., 1998) and thus, by definition, $\left[\mathrm{La}_{\text {MaxClear }}\right]$. Therefore, at steady state, the bioenergetic model seems to be coherent with the experimental observations. Let now turn to the rest-to-work transient phase.

\section{The exercise transient period}

We will consider here the same range of values as defined in the previous section. At the onset of a "square wave" exercise (i.e., a sudden transient phase from rest to a given work load), at the very beginning of the exercise (time $=0$ ), Eq. (3) may be 
written as:

$$
\frac{\dot{w}}{\eta}=\alpha\left[\dot{\mathrm{PC}}_{0}\right]
$$

where $\left[\mathrm{PCr}_{0}\right]$ is the rate of $\mathrm{PCr}$ breakdown at time $=0$. In fact, as it is well known, at the onset of the exercise the Lohman reaction is the fastest and sole ATP regenerative process (Wallimann, 1992). This hypothesis is also compatible with the presence of the so called "glycogen shunt" (Shulman and Rohtman, 2001), a very fast La producing energy yielding pathway. Even in this case, as reported by Shulman and Rohtman (2001), during the first $0-15 \mathrm{~ms}$ only the hydrolysis of PCr participate to [ATP] resynthesis. Of course, oxidative metabolism appears to be too slow to participate to this initial mechanism. Thus, in these conditions, [ATP] does not decrease, not even during the first few milliseconds after the onset of exercise (Chung et al., 1998) and [ATंP] may be considered nill. The term $\dot{w} / \eta$ does not change during the exercise thus, Eq. (17) can be equated with Eq. (8) (the steady state) to obtain:

$$
\alpha\left[\mathrm{PCr}_{0}\right]=\beta\left[\dot{\mathrm{La}}_{s s}\right]+\gamma\left[\dot{\mathrm{O}}_{2 s s}\right]
$$

To intuitively understand the meaning of Eq. (18), one may consider the simple case where the PCr kinetics during the transient is a simple monoexponential (Meyer, 1988; Binzoni et al., 1992; Rossiter et al., 2002):

$$
[\mathrm{PCr}]=\left(\left[\mathrm{PCr}_{0}\right]-\left[\mathrm{PCr}_{s s}\right]\right) e^{-t / \tau_{P C r}}+\left[\mathrm{PCr}_{s s}\right]
$$

where $\left[\mathrm{PCr}_{0}\right]$ is the $\mathrm{PCr}$ resting level, $\tau_{P C r}$ is the exponential time constant of the $\mathrm{PCr}$ splitting rate and $\left[\mathrm{PCr}_{s s}\right]$ is the $\mathrm{PCr}$ at steady state. In this case the term on the left hand side of Eq. (18) may be written as (Binzoni et al., 1994):

$$
-\frac{\alpha}{\tau_{P C r}}\left(\left[\mathrm{PCr}_{0}\right]-\left[\mathrm{PCr}_{s s}\right]\right)=\beta\left[\mathrm{La}_{s s}\right]+\gamma\left[\dot{\mathrm{O}}_{2 s s}\right]
$$

As was the case for $\left[\dot{\mathrm{L}}_{s S}\right]$ and $\left[\dot{\mathrm{O}}_{2 s s}\right]$ (see previous section), $\left(\left[\mathrm{PCr}_{0}\right]-\left[\mathrm{PCr}_{s s}\right]\right)$ at steady state is also linearly related to $\dot{w}$ (Binzoni et al., 1992; di Prampero et al., 2003):

$$
\left[\mathrm{PCr}_{0}\right]-\left[\mathrm{PCr}_{s s}\right]=\alpha_{w} \dot{w}
$$

From Eqs. (9), (17). (20) and (21) may now be written as:

$$
-\frac{\alpha}{\tau_{P C r}} \alpha_{w} \dot{w}=\beta \beta_{w} \dot{w}+\gamma \gamma_{w} \dot{w}
$$

and thus:

$$
\tau_{P C r}=\frac{-\alpha \alpha_{w}}{\beta \beta_{w}+\gamma \gamma_{w}}
$$

This lead to the interesting result that $\tau_{P C r}$ is constant, i.e., independent of the work load $\dot{w}$. From the practical point of view this means that it is possible to compute $\tau_{P C r}$ on the basis of experimental [PCr] data without knowing the exact $\dot{w}$ value. This simplifies for example the collection of NMR data, since it eliminates the tedious problem of proper calibration of the ergometer inside the instrument. Moreover, Eq. (23) describes a physiological "invariant". In addition, by comparing Eq. (13) and Eq. (23) one can see that $\tau_{P C r}$ is related to the efficiency $\eta$. Thus by knowing $\alpha \alpha_{w}$ and $\tau_{P C r}$ it should be possible to estimate $\eta$, a fact that was also pointed out by di Prampero et al. (2003).

If one consider for a while the "old" approximation saying that if the work load is low enough, then the metabolism is "pure" aerobic and we repeat the same calculation as above, then one finds the known relationship (Binzoni et al., 1994):

$$
\tau_{P C r}=\frac{-\alpha \alpha_{w}}{\gamma \gamma_{w}}
$$

In this case, the only difference with Eq. (23) is the term $\beta \beta_{w}$. This means that in the old interpretation $\tau_{P C r}$ represents the speed at which the aerobic glycolysis is "switched-on" at the onset of the exercise is no more valid and must be considered only as an approximation. In fact comparison of Eq. (23) (and Eq. (3)) shows that $\tau_{P C r}$ is the time constant to reach the steady state for both the oxidative and the anaerobic metabolism, considered together. It must be noticed that here the anaerobic metabolism, represented by $\beta \beta_{w}$, is the real $\mathrm{La}$ production by the working muscle and thus completely independent of the "anaerobic threshold" or any intra- or extracellular La recycling mechanism (Brooks, 2000; Brooks, 2002). This means that $\tau_{P C r}$ really represents the time constant of the metabolic activity of the working muscle, and is not influenced by other phenomena.

If $\left[\dot{\mathrm{O}}_{2}\right]$ is also considered to follow an exponential function of time (Rossiter et al., 2002) with the same time constant as [P $\dot{C r}]$, then Eq. (3) implies that $\mathrm{La}^{\cdot}$ is also exponential and that the three terms have the same time constant $\tau_{P C r}$ (i.e., the sum of two exponential function gives a third one if and only if the 3 time constants are equal). This means that measuring $\tau_{P C r}$ we obtain direct informations on the $\left[\dot{\mathrm{O}}_{2}\right]$ and $\mathrm{La}$ kinetics. Concerning $[\mathrm{P} \dot{\mathrm{C}} \mathrm{r}]$ and $\left[\dot{\mathrm{O}}_{2}\right]$ it seems to be the case (Rossiter et al., 2002), i.e. they have the same time constant; concerning $[\mathrm{PC} r]$ and $\mathrm{La}$ it has not yet been demonstrated experimentally.

A last comment has to be made on Eq. (13). In fact, for a given exercise protocol (e.g., cycling on a cycloergometer without changing pedal frequency), Eq. (13) implies that the efficiency $\eta$ does not depend on $\dot{w}$ because $\beta, \beta_{w}, \gamma$ and $\gamma_{w}$, are as a first approximation, and in the range of work loads considered here, all constant. Thus, the number of ATP molecules necessary to produce a unit mechanical work does not depend on $\dot{w}$ (for the $\dot{w}$ range define above in the text). Also this point has to be demonstrated experimentally. 


\section{Conclusion}

The bioenergetic model and the experimental knowledge of the behaviour of the aerobic metabolism and the Lohmann reaction dictates the exact mathematical La-time relationship (in the range from rest to maximal oxygen consumption as defined in the "Theory" section). It appears that La is always produced, even during very low work loads where usually the metabolism is considerd to be "purely" aerobic (we do not consider the rest). The La production rate increases linearly as a function of the work load. Moreover, the anaerobic threshold, is determined by the saturation of the La clearance mechanisms of the body different from the "La shuttle" and not by the involvement of an increased La production at the cellular level when $\dot{w}$ exceed the anaerobic threshold.

It has been also shown, that the half time of the PCr kinetics at the onset of an exercise is dictated both by the speed of aerobic and the anaerobic metabolism, even during very low mechanical power. The time constant of $\mathrm{PCr}$ splitting at work onset does not depend on the workload and represents a physiological invariant. I hope that the present theoretical approach will help to create a common point of view between "old" and "new" generations of scientists and generate new experimental ideas.

Acknowledgements The author would like to thank and acknowledge the support of the Swiss National Science Foundation (grant \#31-58759.99).

\section{References}

Binzoni T (2003) Human skeletal muscle energy metabolism: when a physiological model promotes the search for new technologies. Eur J Appl Physiol 90: 260-269

Binzoni T, Cerretelli P (1994) Bioenergetic approach to transfer function of human skeletal muscle. J Appl Physiol 77: 1784-1789

Binzoni T, Ferretti G, Schenker K, Cerretelli P (1992) Phosphocreatine hydrolysis by 31P-NMR at the onset of constant-load exercise in humans. J Appl Physiol 73: 16441649

Brooks GA (2000) Intra- and extra-cellular lactate shuttles. Med Sci Sports Exerc 32: 790-799

Brooks GA (2002) Lactate shuttles in nature. Biochem Soc Trans 30: 258-264

Chung Y, Sharman R, Carlsen R, Unger SW, Larson D, Jue T (1998) Metabolic fluctuation during a muscle contraction cycle. Am J Physiol 274: C846-C852

Connett RJ, Gayeski TE, Honig CR (1984) Lactate accumulation in fully aerobic, working, dog gracilis muscle. Am J Physiol 246: H120-H128

Cope M, Delpy DT, Wray S, Wyatt JS, Reynolds EO (1989) A CCD spectrophotometer to quantitate the concentration of chromophores in living tissue utilising the absorption peak of water at $975 \mathrm{~nm}$. Adv Exp Med Biol 248: 33-40
Antonutto G, di Prampero P (1995) The concept of lactate threshold. A short review. J Sports Med Phy Fitness 35: 612

Fukuba Y, Walsh ML, Cameron BJ, Morton RH, Kenny CT, Banister EW (1992) The clearance rate of exercise-elevated blood lactate following physical training. Ann Physiol Anthropol 11: 369-376

Mader A, Heck H (1986) A theory of the metabolic origin of “anaerobic threshold”. Int J Sports Med 7 (Suppl 1): 45-65

Margaria R, Cerretelli P, Mangili F (1964) Balance and kinetics of anaerobic energy release during strenuous exercise in man. J Appl Physiol 19: 623-628

Meyer R (1988) A linear model of muscle respiration explains monoexponential phosphocreatine changes. Am J Physiol 254: C548-C553

Myers J, Ashley E (1997) Dangerous curves. A perspective on exercise, lactate, and the anaerobic threshold. Chest 111: 787-795

Di Prampero PE (1981) Energetics of muscular exercise. Rev Physiol Biochem Pharmacol 89: 143-222

Di Prampero PE, Davies CT, Cerretelli P, Margaria R (1970) An analysis of $\mathrm{O} 2$ debt contracted in submaximal exercise. $\mathrm{J}$ Appl Physiol 29: 547-551

Di Prampero PE, Francescato MP, Cettolo V (2003) Energetics of muscular exercise at work onset: the steady-state approach. Pflugers Arch 445: 741-674

Di Prampero PE, Fusi S, Antonutto G (1998) Il concetto di soglia anareobica. Un'analisi critica. Med Sport 51: 393400

Kemp GJ, Radda GK (1994) Quantitative interpretation of bioenergetic data from $31 \mathrm{P}$ and $1 \mathrm{H}$ magnetic resonance spectroscopic studies of skeletal muscle: an analytical review. Magn Reson Q 10: 43-63

Knuttgen HG, Saltin B (1972) Muscle metabolites and oxygen uptake in short-term submaximal exercise in man. J Appl Physiol 32: 690-694

Ross BD, Radda GK, Gadian DG, Taylor D, Bore P, Styles P (1982) Preliminary observations on the metabolic responses to exercise in humans, using 31 phosphorus nuclear magnetic resonance. Ciba Found Symp 87: 145-152

Rossiter HB, Ward SA, Kowalchuk JM, Howe FA, Griffith JR, Whipp BJ (2002) Dynamic asymmetry of phosphocreatine concentration and $\mathrm{O} 2$ uptake between the on- and offtransient of moderate- and high- intensity exercise in humans. J Physiol 541: 991-1002

Shulman RG, Rothman DL (2001) The "glycogen shunt" in exercising muscle: A role for glycogen in muscle energetics and fatigue Proc Natl Acad Sci USA 98: 457-461

Stanley WC, Gertz EW, Wisneski JA, Neese RA, Morris DL, Brooks GA (1986) Lactate extraction during net lactate release in legs of humans during exercise. J Appl Physiol 60: 1116-1120

Stallknecht B, Vissing J, Galbo H (1998) Lactate production and clearance in exercise. Effects of training. A mini-review. Scand J Med Sci Sports 8: 127-131 
Wallimann T, Wyss M, Brdiczka D, Nicolay K, Eppenberger HM (1992) Intracellular compartmentation, structure and function of creatine kinase isoenzymes in tissues with high and fluctuating energy demands: The 'phosphocreatine circuit' for cellular energy homeostasis. Biochem J 281: 2140
Received: September 28, 2004

Accepted: January 20, 2005

Correspondence to: Tiziano Binzoni, PhD, PD, Centre Médical Universitaire, Département de Physiologie, 1211 Genève 4, Switzerland

Phone: +41-22-37-95-358

Fax: +41-22-37-95-402

e-mail: Tiziano.Binzoni@medecine.unige.ch 